\title{
Sample size calculations in pediatric clinical trials conducted in an ICU: a systematic review
}

\author{
Stavros Nikolakopoulos ${ }^{1 *}$, Kit C B Roes ${ }^{1}$, Johanna H van der Lee $^{2}$ and Ingeborg van der Tweel ${ }^{1}$
}

\begin{abstract}
At the design stage of a clinical trial, several assumptions have to be made. These usually include guesses about parameters that are not of direct interest but must be accounted for in the analysis of the treatment effect and also in the sample size calculation (nuisance parameters, e.g. the standard deviation or the control group event rate). We conducted a systematic review to investigate the impact of misspecification of nuisance parameters in pediatric randomized controlled trials conducted in intensive care units. We searched MEDLINE through PubMed. We included all publications concerning two-arm RCTs where efficacy assessment was the main objective. We included trials with pharmacological interventions. Only trials with a dichotomous or a continuous outcome were included. This led to the inclusion of 70 articles describing 71 trials. In 49 trial reports a sample size calculation was reported. Relative misspecification could be calculated for 28 trials, 22 with a dichotomous and 6 with a continuous primary outcome. The median [inter-quartile range (IQR)] overestimation was $6.9[-12.1,57.8] \%$ for the control group event rate in trials with dichotomous outcomes and $-1.5[-15.3,5.1] \%$ for the standard deviation in trials with continuous outcomes. Our results show that there is room for improvement in the clear reporting of sample size calculations in pediatric clinical trials conducted in ICUs. Researchers should be aware of the importance of nuisance parameters in study design and in the interpretation of the results.
\end{abstract}

Keywords: clinical trials, sample size, power, standard deviation, event rate, study design

\section{Review}

Introduction

In randomized controlled trials (RCTs), a priori sample size calculations aim at enrolling sufficient participants to detect a clinically relevant treatment effect. Including too many participants may expose some to an inferior treatment unnecessarily. Including too few may make the likelihood of reaching a definite conclusion too small. The importance of adequate sample size calculations has been widely stressed in the biomedical literature [1-4], including internationally recognized guidelines [5-7]. Most sample size calculations are easily conducted nowadays using specialized software.

In recent years, increasing attention has been given to pediatric RCTs [8-13] for pharmacological interventions due to the fact that many drugs used in children have not (yet) been tested [14]. Drug regulatory agencies

\footnotetext{
* Correspondence: S.N.Nikolakopoulos@umcutrecht.n

'Department of Biostatistics, Julius Center for Health Sciences and Primary Care, University Medical Center Utrecht, Str. 6.131, PO Box 85500, 3508 Utrecht, GA, The Netherlands

Full list of author information is available at the end of the article
}

implemented guidance for sponsors to promote drug research in children, leading to more trials being designed and conducted [15]. Recruitment difficulties $[16,17]$ and ethical considerations $[18,19]$ make pediatric trials more challenging, especially with critically ill children, e.g. children being treated in ICUs. In such cases, the importance of a rigorously designed RCT is stressed.

In the design phase of an RCT, the sample size is calculated based on the primary outcome variable. Sample size depends on parameters that are estimated or assumed, in addition to the set criteria of type I error and power. In addition to the clinically relevant treatment effect to be detected, assumptions need to be made about so-called nuisance parameters (NPs). A NP is a parameter that is not of direct interest but must be accounted for in the analysis of the treatment effect and thus also in the sample size calculation. Examples of NPs are the event rate in the control group (control group event rate, CER) when the clinical outcome of interest is dichotomous and the standard deviation (SD, assumed equal across groups), when the clinical outcome is continuous. 
The value of a NP substantially affects the sample size calculation; therefore the value assumed should be as reliable as possible. Of course, the observed value once the trial is completed will differ from the assumed value at the design stage. If the assumed value is different from the (unknown) population value, we refer to it as the misspecification of the nuisance parameter. Misspecification can have serious consequences for the actual power of the trial and the smallest possible effect size that can be detected.

When a sample size calculation is performed, the value of the NP used corresponds to its assumed population value. Therefore, misspecification can be shown on a per trial basis in terms of statistical significance. That is, test whether the observed value is significantly different from the assumed population value. However, our focus will be on systematic misspecification. We are interested in exploring whether there is systematic over- or underestimation of NPs in a specific population of pediatric RCTs, and what the consequences of such a systematic misspecification are on the design aspects of these RCTs and the inference that can be drawn from them. There are various ways to arrive at an assumption about the value of a NP. One can estimate it based on data from earlier trials or other types of studies, or conduct a pilot study. However, all these methods can lead to misspecification of the NP [20-24].

Previous research has shown that RCTs in general use sample sizes that are too small due to unduly optimistic a priori assumptions [22]. This optimism is partly reflected in the assumed clinically relevant treatment effect, but can also occur as a direct effect of misspecifying a NP. For example, the value of the risk ratio (RR), which is the event rate in the experimental group divided by the event rate in the control group, is directly dependent on the event rate in the control group, which has to be estimated before the start of the trial. Similarly, for a continuous outcome, the value of the SD determines how large the difference in means is [25]. For instance, in a sample of 100 patients per arm, a difference of 10 units in some continuous measurement would be significant $(P=0.047)$ if the SD was equal to 30 . If the SD was 40 , this difference would no longer be statistically significant $(P=0.11)$.

As an illustrative example of an RCT with a dichotomous endpoint, let us consider a clinical trial comparing the efficacy of low-dose dexamethasone with a saline placebo in ventilator-dependent infants. The clinical endpoint in this case could be survival free of major neurosensory disability after 2 years. In this case, the NP is the CER. If a CER of $50 \%$ is assumed in the design stage, a total sample size of $n=334$ (167 per group) would be required to detect a RR of 1.3 with a $5 \%$ two-sided type I error level and $80 \%$ power. However, if the true CER is $35 \%$ instead of $50 \%$, the required sample size to detect the same effect size, i.e. $R R=1.3$, would be 678 (339 per group). Figure 1 shows how large the differences in required sample size can be for relatively small differences in the true CER, for RRs of $1.2,1.3$ and 1.5.

When comparing two groups with respect to a dichotomous outcome, the absolute risk difference is customarily used in sample size calculations as the effect size that is considered clinically relevant. The absolute risk difference is easier to interpret for clinical purposes, since it can be translated into a number needed to treat. However, for our present research we consider the RR to be a more consistent way to compare the efficacy of two treatments regardless of the CER; its value represents a relative measure of difference, taking into account the level of efficacy in the control group. For example, one could argue it is not logical to expect the same absolute difference, e.g. $20 \%$, if the CER is $50 \%$ or $30 \%$.

There is published research addressing the accuracy and quality of sample size calculations and their reporting in clinical trials [22,26,27]. These papers reported several discrepancies between protocols and reports [26] but also inadequate reporting and inaccuracies in general [27]. Important guidelines for the reporting of RCTs are the CONSORT statement [5] and the statement from the International Committee of Medical Journal Editors (ICMJE) on clinical trial registration [7]. Reporting of sample size calculations would be expected to have improved, since it is explicitly required by these statements.

Besides the above mentioned papers, which cover the general spectrum of sample size calculation in RCTs, little is known about the misspecification of NPs in pediatric RCTs in particular. To investigate the impact of systematic misspecification of NPs in pediatric RCTs, we reviewed published papers reporting results of pediatric RCTs. We focused on trials conducted in neonatal intensive care units and pediatric intensive care units (PICUs) due to the vulnerability of the target populations in such studies. We furthermore focused on trials evaluating pharmacological interventions because of the increased interest from regulators and the ethical considerations mentioned above. These aspects require a high standard of clinical trial design. Finally, we will provide guidance about what can be done to prevent misspecification and its consequences.

\section{Search strategy}

We searched MEDLINE through PubMed, following the sensitivity- and precision-maximizing search strategy for identifying RCTs as suggested by the Cochrane Handbook for Systematic Reviews of Interventions [28]. We searched for papers between 1 January 2006 and 31 October 2011, which covers a 5-year span from the application of the clinical trial registration statement from the ICMJE. Further limits imposed were 'Humans' for species, 'English' for language and 'All Child: 0-18 years' for 


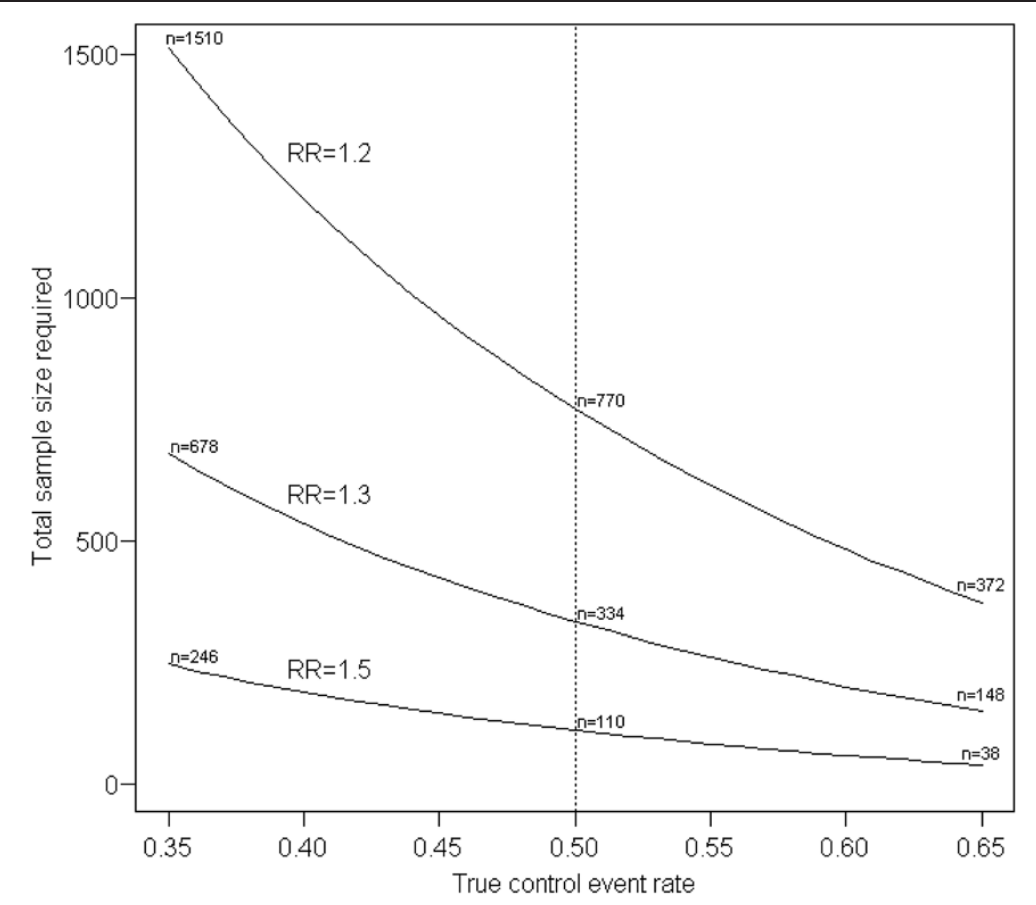

Figure 1 Impact of misspecification of control event rate on total sample size. The sample sizes required to detect RRs with $80 \%$ power at a $5 \%$ two-sided type I error level are shown. The dotted vertical line indicates the assumed CER. (See text for further explanation). CER, control group event rate; RR, risk ratio.

age. Additional keywords 'Intensive care,' 'ICU,' 'PICU' or 'NICU' were used.

\section{Selected articles}

Selection and data extraction were performed by two authors (SN and IvdT) independently. Disagreements were discussed to reach consensus. Selection was restricted to publications concerning two-arm parallel group RCTs where efficacy assessment was the main objective. We only included trials with pharmacological interventions. Only trials with a dichotomous or a continuous outcome were included. Trials that were specifically described as Phase I or II, pilot or exploratory were excluded. We excluded trials that were designed with more than two groups (e.g. factorial designs and dose-response trials).

\section{Data extraction}

General characteristics of each study, namely, year of publication, included patients, experimental and control interventions, primary outcome, type of primary outcome (dichotomous/continuous), registration (yes/no and if yes, registration code) and use of a crossover design were extracted. For the a priori sample size calculations, the following information was extracted: type I error, power, one- or two-sided testing, the assumed value of NPs (since we only considered dichotomous and continuous outcomes, the NPs recorded were the assumed CER and common SD, respectively), expected effect size (i.e., the standardized effect size, expressed as Cohen's $d$ for continuous outcomes, which is the mean difference between the two groups divided by the common standard deviation, and the risk ratio for dichotomous outcomes), the required sample size (with and without accounting for dropout, if applicable) and, if reported, the information source on which the assumptions concerning the NP were based, e.g. literature, own experience or pilot study. From the results sections of the articles, we extracted information on the actual sample size randomized, the one used in the analysis (irrespective of whether an intention-totreat or per-protocol analysis was conducted), the observed value of the NP and the observed effect size.

Some papers were included in this review because the outcomes measured were continuous or dichotomous, but it was not made clear, either in the sample size calculations or in the text, which outcome was the primary one. In these cases, the primary outcome type was coded as 'unclear'. For a trial to be considered as reporting an a priori sample size calculation, at least the power should have been mentioned in the methods section of the publication. When the type I error was not reported, a value of 0.05 (two-sided) was assumed. The reported assumed NP value was taken into consideration when it was explicitly mentioned or traceable from a cited publication; thus, we did not attempt to (re-) calculate the assumed NP from the information provided in the methods section of the article. 


\section{Data analysis}

Two authors (SN and IvdT) replicated the sample size calculations independently, based on the assumed parameters. These replicated sample sizes were calculated based on Student's $t$-test for continuous variables and based on the chi-square test for dichotomous variables, which is equivalent to the two-sample binomial test ( $Z$ test). We also recalculated required sample sizes based on the empirical values of the NPs as published in the paper. For a continuous outcome for which median and range were reported instead of mean and $\mathrm{SD}$, the $\mathrm{SD}$ was calculated according to Hozo et al. [29].

We also calculated the minimum detectable effect size (MDES), given the sample size available for analysis and the observed NP (Figure 2). Note that this quantity is different from the effect size expected for the a priori sample size calculation. MDES reflects the minimum difference that would yield a significant result for a given sample size, NP and type I error level; this effect size will be smaller than the effect size on which the sample size calculation is based, i.e. the clinically relevant effect size. MDES has a power of $50 \%$ to be detected if it is true, therefore for any trial designed with more than 50\% power for a clinically relevant effect size, the MDES will be smaller than the latter [30].
The per trial estimate of misspecification of the NP was calculated relative to the observed NP as:

\section{(Assumed NP - Observed NP)/Observed NP.}

The relative difference for the MDES was calculated in the same way:

(MDES with the assumed NP - MDES with the observed NP)/ MDES with the observed NP.

As mentioned before, empirically obtained estimators of nuisance parameters are also subject to random variation; therefore any systematic trend in the direction of possible misspecifications was our main interest. Statistical analyses were conducted with $\mathrm{R}$, version 2.13.1 and SPSS (PASW statistics) version 17.

\section{Results}

The search yielded 742 citations. After screening titles and abstracts, 112 articles were selected for full-text appraisal. Of these 112 articles, 70 complied with our inclusion criteria. One of the trials reported two distinct subgroups for which a different power calculation and hypothesis were described, and they were taken into account as two distinct trials for all the analyses except the registration rate, leading to a total of 71 trials described in 70 papers (see Figure 3).

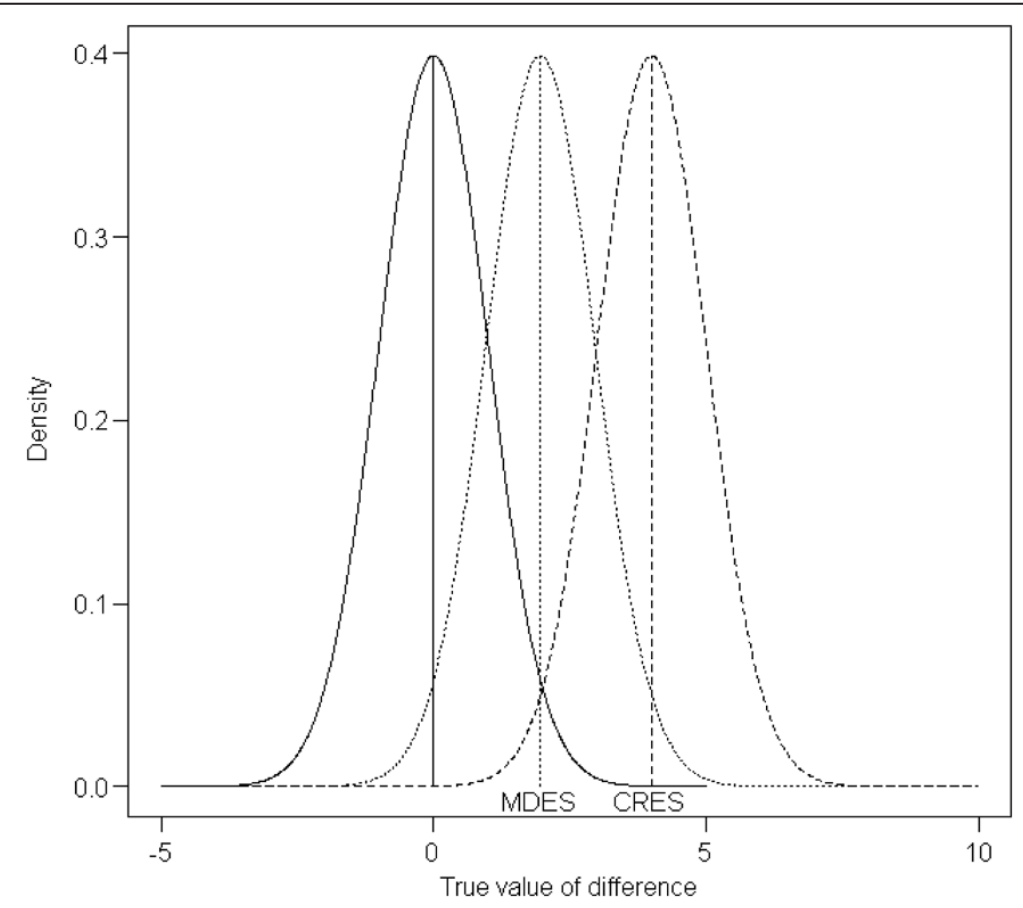

Figure 2 Illustration of minimum detectable effect size. The minimum detectable effect size (MDES) is the minimum difference between groups that yields a statistically significant result. The power of the study is calculated using the clinically relevant effect size (CRES). The density of the left curve to the right of the MDES represents the type I error, i.e. $2.5 \%$ (one-sided) in this case. The density of the middle curve to the right of the MDES represents the power for the MDES to be detected, i.e. 50\%. The density of the right curve to the right of the MDES represents the power for the CRES to be detected, i.e. the power of such a study, which is $98 \%$ in this example. CRES, clinically relevant effect size; MDES, minimum detectable effect size. 

studies, studies in adults etc.)

Figure 3 Flowchart of the search and inclusion procedure.

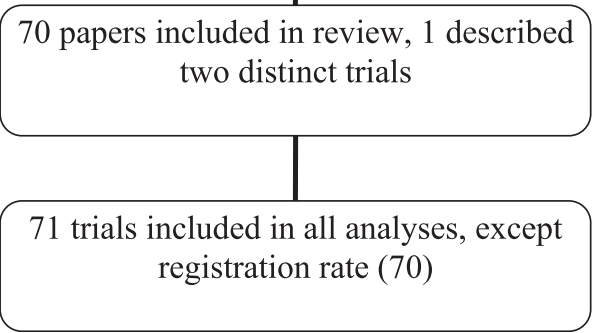

112 Papers selected for full-text appraisal

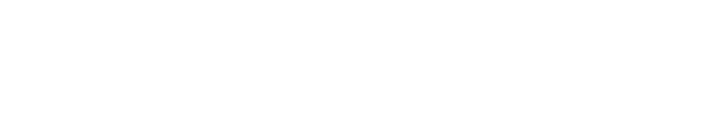

71 trials included in all analyses, except registration rate $(70)$

One trial had a crossover design with a dichotomous outcome and used the McNemar test for sample size calculation as well as for analysis. Therefore, this study was included in the analysis concerning misspecification of NPs but not in recalculation of sample and effect sizes, because even though the NP of the McNemar test is still a proportion (of discordant pairs), the effect size is not comparable with the one in a chi-square test.

The PRISMA checklist can be found in Additional file 1, the reviewed articles in Additional file 2 and the extracted data in Additional file 3.

\section{Descriptive characteristics}

Table 1 summarizes the basic characteristics of the included trials. Of the 71 trials, 31 were performed for preterm or term neonates. The information concerning the sample size calculations is shown in Table 2. Of the 12 trials reporting on-line registration, 3 (25\%) were registered in the International Standard Randomized Controlled Trial Number Register (ISRCTN) and 9 (75\%) were registered in ClinicalTrials.gov (NCT). In 11 of the 49 papers (22\%) that reported a sample size calculation in the methods section, a different analysis was used to evaluate the result of
Table 1 Basic characteristics of the 70 included papers

\begin{tabular}{ll}
\hline Characteristic & $\mathbf{N}(\%)$ \\
\hline Registration & $12(17)$ \\
$\quad$ Registration reported & \\
Study population & $31(44)$ \\
$\quad$ Neonates (0 to 1 years old) & $24(34)$ \\
$\quad$ Children (>1 years old) & $15(21)$ \\
Both & \\
Intervention in the control group & $30(43)$ \\
$\quad$ Placebo & $35(50)$ \\
Active control & $5(7)$ \\
Standard care/none & \\
Funding source & $19(27)$ \\
Public & $6(9)$ \\
Private & $45(64)$ \\
$\quad$ Not clear &
\end{tabular}


Table 2 Characteristics of sample size calculations of the 71 included trials (70 papers)

\begin{tabular}{|c|c|}
\hline Characteristic & $N(\%)$ \\
\hline \multicolumn{2}{|l|}{ Type of primary outcome } \\
\hline Dichotomous & $30(42)$ \\
\hline Continuous & $39(55)$ \\
\hline Unclear & $2(3)$ \\
\hline \multicolumn{2}{|l|}{ A priori sample size calculation } \\
\hline At least power reported & $49(69)$ \\
\hline \multicolumn{2}{|l|}{$\rightarrow$ Of these } \\
\hline NP reported & $35(71)$ \\
\hline NP reported as proportion of total & $35(49)$ \\
\hline No report of a priori sample size calculation & $22(31)$ \\
\hline \multicolumn{2}{|l|}{ Information source of NP assumption } \\
\hline \multicolumn{2}{|l|}{$\rightarrow$ Of the 49 trials that report at least power } \\
\hline Literature & $21(43)$ \\
\hline Own experience & $9(18)$ \\
\hline Pilot study & $6(12)$ \\
\hline Not reported & $13(27)$ \\
\hline \multicolumn{2}{|c|}{$\rightarrow$ Of the 35 trials that report an assumed NP value } \\
\hline Literature & $14(40)$ \\
\hline Own experience & $8(23)$ \\
\hline Pilot study & $6(17)$ \\
\hline Not reported & $7(20)$ \\
\hline
\end{tabular}

the trial than the one assumed in the sample size calculation. Four of these applied nonparametric analyses, presumably due to a failure to meet the assumptions of the parametric tests and/or unfamiliarity with sample size calculations for non-normally distributed outcomes.

In the reports of all 12 registered trials, an a priori sample size calculation (at least power mentioned) was reported; this was the case in 35 reports out of 58 trials (60\%) that did not report a registration. The rate of reporting the expected NP was 10 out of 12 for papers that reported registration (83\%) and 24 out of $58(41 \%)$ for papers that did not. Note that for these figures the number of papers is the total sample size (70) rather than the number of trials (71).

\section{Misspecification of nuisance parameters}

Seven of the 35 articles report an assumed value for the NP, but did not report the observed value for this parameter in the results section, because a different analysis was conducted (e.g. nonparametric testing). Thus, we were able to calculate the relative misspecification for 28 trials, 22 with a dichotomous and 6 with a continuous primary outcome. The results are summarized in Figure 4. As can be seen, CERs were more often overestimated than underestimated; SDs were more often underestimated. The median [inter-quartile range (IQR)] overestimation was $6.8[-12.1,57.8] \%$ for trials with dichotomous outcomes and $-1.5[-15.3,5.1] \%$ for trials with continuous outcomes. As shown in the example above, overestimation of the control event rate can have severe consequences for the sample size requirements. It is indicative that the total estimated sample size for the subgroup of trials with continuous outcomes was 846 subjects though it should have been 1,044 subjects, while for the ones with dichotomous outcomes, in total 3,326 subjects were planned to be enrolled, while 3,904 should have been enrolled based on the values for the NP actually observed.

The effect of the misspecification of the NP was apparent on the average power of the studies reviewed. The average power required by design was $83 \%$ while the average power taking the observed NPs into account, based on the sample sizes calculated in the papers, would have been $73.9 \%$. Based on our replicated sample sizes the power achieved would be $71.8 \%$. However, these results should only be taken as indicative and exploratory, as we share the concerns of other authors about power calculations after data is collected [31]. More specifically, researchers should be very careful with interpreting the post-hoc power, which is the power calculated for the observed treatment effect, and the same applies to the observed NP. There was no evidence of a relation between the source used to make assumptions for the NP and the magnitude of misspecification.

\section{Minimum detectable effect size in trials with dichotomous outcomes}

Relative differences between the MDES based on a priori assumptions and the MDES based on the observed values of the NPs are presented in Figure 5. The proportion of trials in which the observed MDES was larger than the assumed MDES was roughly equal to the proportion of trials in which it was smaller (the median misspecification was $1 \%$ ). However, the distribution of the discrepancies is skewed (minimum $=-30 \%$, $\max -$ imum $=224 \%$, IQR $=-7 \%$ to $23 \%$ ), with some extreme values, reflecting the skewed distribution of the discrepancies in the NPs. This shows that the misspecification of NP more often causes MDES to be smaller than anticipated by design, therefore making significant findings less probable.

\section{Discussion}

In this review of 71 pediatric clinical trials, our main goal was to assess the presence and magnitude of systematic misspecification of NPs in sample size calculations. Deviations between assumed and realized values of NPs can lead to undesirable trial characteristics like underestimated sample size and overestimated power. This can possibly lead to important clinical improvements being missed and 


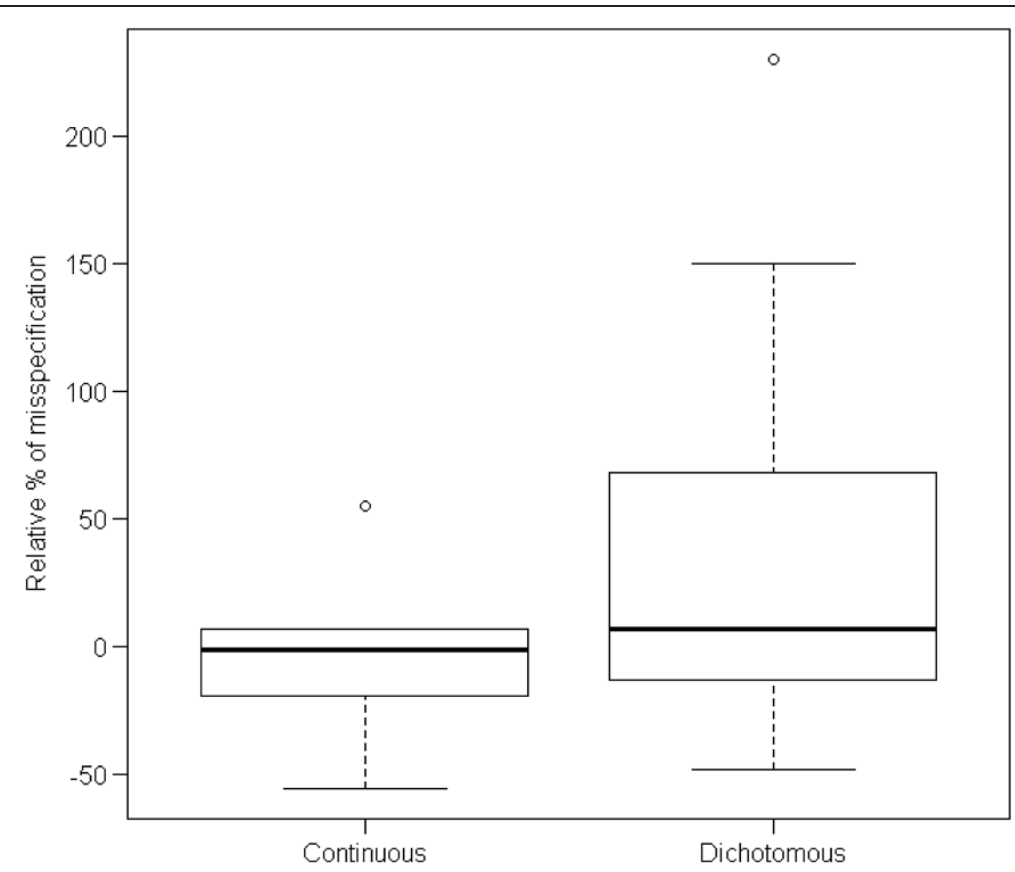

Figure 4 Misspecification of nuisance parameters. Relative misspecification of NPs in the trials reviewed = (Expected value of NP - Observed value of NP)/Observed value of NP.

to an increased number of trials unnecessarily considered negative or failures. It also reduces the value of individual patients participating in clinical trials. Some experts consider underpowered trials to be unethical [32].

Of course, observed parameter values deviate from the assumed ones, due to random fluctuation and this is incorporated in sample size estimation. If estimation is accurate, it is expected that these discrepancies will take place in both directions (both over- and underestimating), causing no overall effect in the design characteristics of the RCTs reviewed. However, as the results of our review show, there is systematic misspecification of

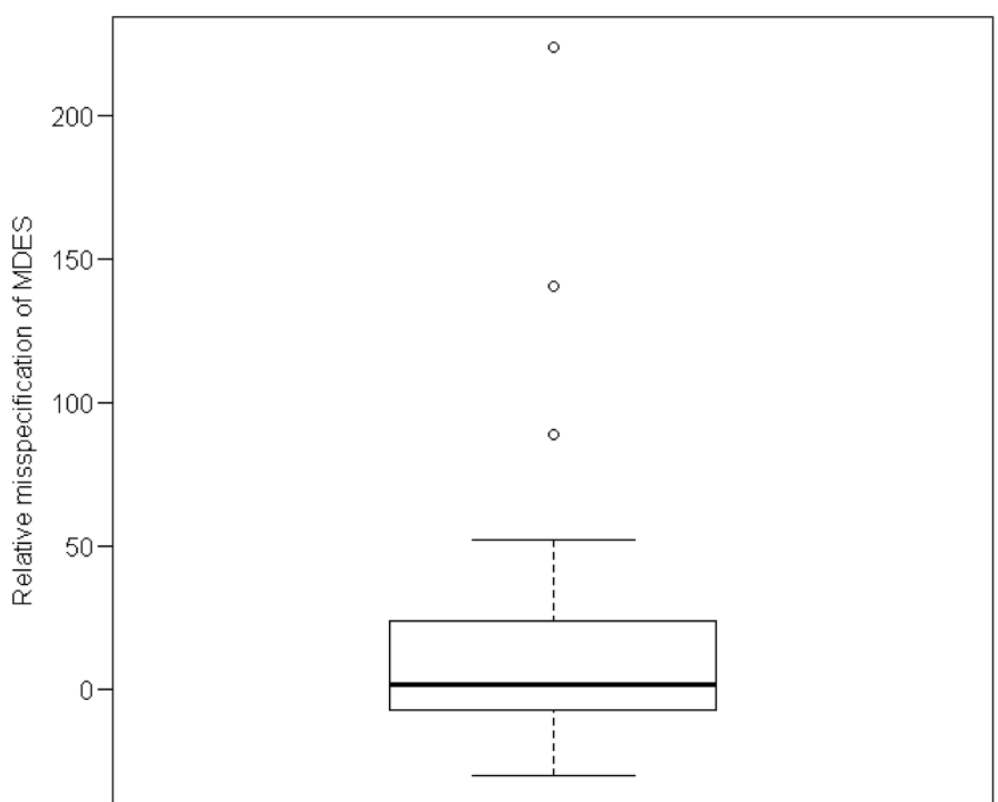

Figure 5 Relative misspecification (as a percentage) of MDES in trials with a dichotomous outcome. The vertical axis represents the quantity: (MDES with the assumed NP - MDES with the observed NP)/MDES with the observed NP. MDES, minimum detectable effect size; $N P$, nuisance parameter. 
nuisance parameters, resulting in about $10 \%$ lower average power of the studies than required in the design stage. As a result, more patients should have been studied for the conclusions of the studies to be in compliance with their design characteristics. The loss in power theoretically results in $10 \%$ of studies with promising interventions being expected to conclude incorrectly that there is no benefit.

An important issue of concern is that reporting of sample size calculations is still not adequate. This is in accordance with the findings by Charles et al. [27]. We assumed that the CONSORT statement and the clinical trials registration would have led to more transparent reporting, but the percentage of registered trials was very low (17\%). It should be noted though that while trial registration was stated as a requirement for publication by ICMJE, we did not restrict our search to these journals. The rate of registration may in reality have been higher, since our information depended on explicit reporting in journal articles.

Misspecification of the NP has more severe consequences for trials with a dichotomous outcome than for those with a continuous outcome. As the results of our review show, the CER was found to be up to $200 \%$ misspecified. One way around this is to avoid dichotomizing continuous outcomes, if possible, and also to avoid treating time-to-event outcomes as binary. Misspecification, especially underestimation, of the SD for a trial with a continuous outcome also has considerable consequences. We are unable to draw reliable conclusions from our study, because of the very small number of trials with a continuous endpoint reporting both assumed and realized values of the standard deviation.

Further limitations of our study are the quite specific inclusion criteria (trials with pharmacological interventions and conducted in an ICU). The findings may not be generalizable beyond this group of trials. Additionally, RCTs that are not analyzed by the intention-to-treat principle are likely to introduce bias in estimation of the treatment effect, which could also have implications for sample size calculations. However, it was seldom reported whether the trial was analyzed by the intention-to-treat principle (in only 19 papers was it clearly stated). Furthermore, even though the search was conducted in a systematic way, the possibility that some trials that could fit our inclusion criteria were missed, cannot be excluded. However, we do not expect this to affect the validity of our results since the scope of this review is to explore the state of affairs rather than, e.g., evaluate the effectiveness of an intervention where missing a trial would be considered a caveat.

Misspecification of NPs occurs frequently in pediatric clinical trials conducted in ICUs. Failure of reporting a priori assumptions about NPs appeared to be more common in the trial reports included in this review than in trials published in high-impact medical journals [27], even though these trials included an extremely vulnerable population. Awareness should be raised of this matter and journal editors should be more demanding concerning reporting standards adopted by the highimpact journals.

Methodologies exist that are less sensitive to assumptions of NPs, such as using a more flexible design and analysis (e.g. sequential trials) or re-estimation of the sample size (internal pilot). Another way would be to state the expectations for the clinically relevant effect size in a standardized way (e.g. use of Cohen's standardized effect size, [25,33]). This allows one not to make specific assumptions for the NP but rather state the magnitude of the effect size considered clinically relevant (e.g. small, medium or large effect size).

\section{Conclusions}

Research in vulnerable populations, like children, is challenging and demanding. Cumulative knowledge is difficult to acquire but necessary for evidence-based evaluation of medical interventions. This should be done in the most efficient and ethical way possible and a well-thought-out study design is a crucial step towards this goal. We would strongly advise editors of all medical journals to adopt the reporting standards guidance and be more demanding that authors conform to these standards.

\section{Additional files}

Additional file 1: PRISMA checklist of the systematic review.

Additional file 2: Articles reviewed.

Additional file 3: Data extracted from the reviewed articles.

\section{Abbreviations}

CER: control group event rate; CRES: clinically relevant effect size; ICMJE: International Committee of Medical Journal Editors; IQR: inter-quartile range; MDES: minimum detectable effect size; NP: nuisance parameter; PICU: pediatric intensive care unit; RCT: randomized controlled trial; RR: risk ratio; SD: standard deviation.

\section{Competing interests}

The authors declare that they have no competing interests.

\section{Authors' contributions}

SN conducted the literature search, study selection and data extraction; carried out the analyses; drafted and revised the manuscript; and approved the final manuscript as submitted. KCBR conceptualized the study, reviewed the manuscript and approved the final manuscript as submitted. JHvdL reviewed the manuscript, extracted relevant medical information and approved the final manuscript as submitted. IvdT conceptualized the study, selected the studies and extracted data, reviewed the analyses, reviewed the manuscript and approved the final manuscript as submitted.

\section{Acknowledgements}

This research was partly funded by the Netherlands Organization for Health Research and Development (ZonMW) through grant number 152002035, for 'Optimal design and analysis for clinical trials in orphan diseases'. 


\section{Author details}

'Department of Biostatistics, Julius Center for Health Sciences and Primary Care, University Medical Center Utrecht, Str. 6.131, PO Box 85500, 3508 Utrecht, GA, The Netherlands. ${ }^{2}$ Clinical Research Unit, Woman-Child Center, Academic Medical Center, University of Amsterdam, Amsterdam, The Netherlands.

Received: 21 January 2014 Accepted: 24 June 2014

Published: 8 July 2014

\section{References}

1. Noordzij M, Tripepi G, Dekker FW, Zoccali C, Tanck MW, Jager KJ: Sample size calculations: basic principles and common pitfalls. Nephrol Dial Transplant 2010, 25:1388-1393.

2. Schulz KF, Grimes DA: Sample size calculations in randomised trials: mandatory and mystical. Lancet 2005, 365:1348-1353.

3. Eng J: Sample size estimation: how many individuals should be studied? Radiology 2003, 227:309-313.

4. Halpern SD, Karlawish JH, Berlin JA: The continuing unethical conduct of underpowered clinical trials. JAMA 2002, 288:358-362.

5. Schulz KF, Altman DG, Moher D, CONSORT Group: CONSORT 2010 statement: updated guidelines for reporting parallel group randomised trials. BMJ 2010, 340:C332.

6. Harmonised $\mathrm{ICH}$, Tripartite Guideline: Statistical principles for clinical trials: International Conference on Harmonisation E9 expert working group. Stat Med 1999, 18:1905-1942.

7. De Angelis CD, Drazen JM, Frizelle FA, Haug C, Hoey J, Horton R, Kotzin S, Laine C, Marusic A, Overbeke AJ, Schroeder TV, Sox HC, Van Der Weyden $M B$ : Is this clinical trial fully registered? - A statement from the International Committee of Medical Journal Editors. N Engl J Med 2005, 352:2436-2438.

8. Pasquali SK, Lam WK, Chiswell K, Kemper AR, Li JS: Status of the pediatric clinical trials enterprise: an analysis of the US ClinicalTrials.gov registry. Pediatrics 2012, 130(5):1269-1277.

9. Hartling L, Wittmeier KD, Caldwell P, van der Lee H, Klassen TP, Craig JC, Offringa M, StaR Child Health Group: StaR child health: developing evidence-based guidance for the design, conduct, and reporting of pediatric trials. Pediatrics 2012, 129(Suppl 3):S112-S117.

10. Wittmeier KD, Craig J, Klassen TP, Offringa M: The mission of StaR Child Health is to improve the quality of the design, conduct, and reporting of pediatric clinical research by promoting the use of modern research standards. Intro Pediatr 2012, 129(Suppl 3):S111.

11. Caldwell PH, Murphy SB, Butow PN, Craig JC: Clinical trials in children. Lancet 2004, 364:803-811.

12. Klassen TP, Hartling L, Craig JC, Offringa M: Children are not just small adults: the urgent need for high-quality trial evidence in children. PLoS Med 2008, 5:e127

13. Food and Drug Administration: International conference on harmonisation; guidance on E11 clinical investigation of medicinal products in the pediatric population; availability: notice. Fed Regist 2000 65:78493-78494

14. Conroy S, McIntyre J, Choonara I, Stephenson T: Drug trials in children: problems and the way forward. Br J Clin Pharmacol 2000, 49:93-97.

15. Bosch X: Pediatric medicine: Europe follows US in testing drugs for children. Science 2005, 309:1799.

16. Caldwell PH, Butow PN, Craig JC: Parents' attitudes to children's participation in randomized controlled trials. J Pediatr 2003, 142:554-559.

17. Sureshkumar P, Caldwell P, Lowe A, Simpson JM, Williams G, Craig JC: Parental consent to participation in a randomised trial in children: associated child, family, and physician factors. Clin Trials 2012, 9:645-651.

18. Laventhal N, Tarini BA, Lantos J: Ethical issues in neonatal and pediatric clinical trials. Pediatr Clin North Am 2012, 59:1205-1220.

19. Gill D, Ethics Working Group of the Confederation of European Specialists in Paediatrics: Ethical principles and operational guidelines for good clinical practice in paediatric research. Recommendations of the Ethics Working Group of the Confederation of European Specialists in Paediatrics (CESP). Eur J Pediatr 2004, 163:53-57.

20. van der Lee JH, Tanck MW, Wesseling J, Offringa M: Pitfalls in the design and analysis of paediatric clinical trials: a case of a 'failed' multi-centre study, and potential solutions. Acta Paediatr 2009, 98:385-391.
21. Proschan MA: Two-stage sample size re-estimation based on a nuisance parameter: a review. J Biopharm Stat 2005, 15:559-574.

22. Vickers AJ: Underpowering in randomized trials reporting a sample size calculation. J Clin Epidemiol 2003, 56:717-720.

23. Kraemer HC, Mintz J, Noda A, Tinklenberg J, Yesavage JA: Caution regarding the use of pilot studies to guide power calculations for study proposals. Arch Gen Psychiatry 2006, 63:484-489.

24. Sim J, Lewis M: The size of a pilot study for a clinical trial should be calculated in relation to considerations of precision and efficiency. J Clin Epidemiol 2012, 65:301-308.

25. Cohen J: A power primer. Psychol Bull 1992, 112:155-159.

26. Chan AW, Hrobjartsson A, Jorgensen KJ, Gotzsche PC, Altman DG: Discrepancies in sample size calculations and data analyses reported in randomised trials: comparison of publications with protocols. BMJ 2008, 337:a2299.

27. Charles P, Giraudeau B, Dechartres A, Baron G, Ravaud P: Reporting of sample size calculation in randomised controlled trials: review. BMJ 2009, 338:b1732.

28. The Cochrane collaboration. [http://www.cochrane-handbook.org]

29. Hozo SP, Djulbegovic B, Hozo I: Estimating the mean and variance from the median, range, and the size of a sample. BMC Med Res Methodol 2005, $5: 13$.

30. Chuang-Stein C, Kirby S, Hirsch I, Atkinson G: The role of the minimum clinically important difference and its impact on designing a trial. Pharm Stat 2011, 10:250-256

31. Hoenig JM, Heisey DM: The abuse of power: the pervasive fallacy of power calculations for data analysis. Am Statistician 2001, 55:19-24.

32. Altman DG: The scandal of poor medical research. BMJ 1994, 308:283.

33. van der Tweel I, Askie L, Vandermeer B, Ellenberg S, Fernandes RM, Saloojee H, Bassler D, Altman DG, Offringa M, Van der Lee JH, for the StaR Child Health Group: Standard 4: determining adequate sample sizes. Pediatrics 2012, 129(Suppl 3):S138-S145.

doi:10.1186/1745-6215-15-274

Cite this article as: Nikolakopoulos et al: Sample size calculations in pediatric clinical trials conducted in an ICU: a systematic review. Trials $201415 \cdot 274$

\section{Submit your next manuscript to BioMed Central and take full advantage of:}

- Convenient online submission

- Thorough peer review

- No space constraints or color figure charges

- Immediate publication on acceptance

- Inclusion in PubMed, CAS, Scopus and Google Scholar

- Research which is freely available for redistribution 